\title{
IS CHRONIC KIDNEY DISEASE REVERSIBLE?
}

\author{
Sergiu Dumitrache, Mircea Penescu
}

\begin{abstract}
Chronic kidney disease, no matter the aetiology, has a progressive evolution and a negative prognosis. The rapidity of nephrons loss and deterioration of renal function depends on the aetiology of the kidney disease, the prompt diagnosis, efficiency of the treatment and the patient compliance to the treatment, diet and suitable lifestyle. The therapeutic control of the mechanisms of progression have also demonstrated that it could be possible to attenuate or even to stop the evolution towards kidney failure.
\end{abstract}

Keywords: chronic kidney disease, immunological mechanisms, renin angiotensin aldosterone system.

\section{Rezumat}

Boala cronică de rinichi, indiferent de etiologie, are o evoluție progresivă și un prognostic negativ. Rapiditatea pierderii nefronilor și a deteriorării funcției renale depinde de etiologia bolii renale, diagnosticul prompt, eficiența tratamentului și respectarea de către pacient a tratamentului, dietei și stilului de viață adecvat. De asemenea, controlul terapeutic al mecanismelor de progresie a bolii a demonstrat că ar putea fi posibilă atenuarea sau chiar stoparea evoluției spre insuficiența renală.

Cuvinte cheie: boală cronică de rinichi, mecanisme imunologice, sistem renină angiotensină aldosteron.

Chronic kidney disease, no matter the aetiology, has a progressive evolution and a negative prognosis. The rapidity of nephrons loss and deterioration of renal function depends on the aetiology of the kidney disease, the prompt diagnosis, efficiency of the treatment and the patient compliance to the treatment, diet and suitable lifestyle.

However, we can identify certain therapeutically measures for preservation or even recovery of the renal function. There are arguments for rehabilitation in CKD, contrary to the general perception until this moment. Here are some examples:

In patients with IgA glomerulonephritis, the treatment with high doses of inhibitory angiotensin converting enzyme significantly reduces the fibrotic lesions. Similarly, in diabetic patients with diabetic nephropathy the pancreas transplant determine 


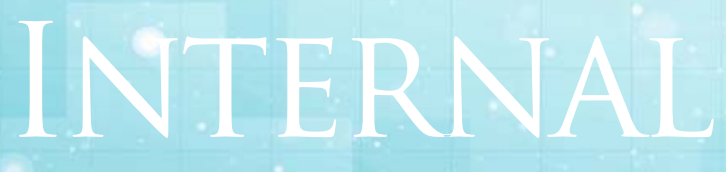

General Reviews

regression of the lesions. These observations contradict the fact that beginning from a certain point of renal disease, the mechanisms of self promoting lesions lead to progressive deterioration of renal function until the need of function substitution.

The progressive and predictive decline of renal function represents the fundamental concept in CKD. The linear evolution meaning a relatively constant rate of functional degradation suggests the action of certain compensatory physiopathologycal mechanisms that will become themself the cause of progression of renal lesions. This central paradigma implies two other aspects. First, accelerated evolution of kidney disease could be attributed to new succesivly acting acute renal lesions. It isn't very clear if CKD can be attributed to successive subclinical acute renal lesions, at least in the case of patients that didn't have a known renal disease. So appreciating the degradation rhythm of renal function represents the tool for monitoring any acute lesions superimposed on the kidney.

Secondly, there is another asspect implied by the progressive evolution of CKD on many years. The physiopathological mechanisms involved in progression of renal failure act on progressive reduced number of nephrons and so the evolution would be selfaccelerated. Therapeutic strategies attenuates or cancel the accelerated evolution rhythm of the disease. In addition, the new physiopathological mechanism induced by hypertension and metabolic disturbancies, like acidosis, hyperphosphatemia and hypocalcemia with consequences on mineral metabolism and aggravation of vascular disease, would favor the additional acceleration of kidney disease in the absence of the treatments.

The regressive evolution of CKD in patients receiving treatment and, probably, in some cases, even spontaneously, supports the idea of potentially reversibility of morphological lesions. The mechanisms through which renal lesions are induced are immunological and non-immunological. Immunological mechanisms are responsible for acute kidney lesions, while nonimmunological mechanisms are associated with the development and slow progression of chronic kidney lesions.

The inflammation characteristic of immunological mechanisms is the one that leads to fibrosis and sclerosis. On the other hand, non-immunological mechanisms are associated with degenerative changes which lead to fibrosis and sclerosis.

Non-immunological mechanisms are of adaptive type associated with the reduction in the number of nephrons, hypertrophy of remaining nephrons, glomerular hypertension and hyper filtration, proteinuria, but also high protein intake and 
dyslipidemia. The process of fibrosis implies the deposition of extracellular matrix which replaces the normal structure of the nephron. The fibrosis and sclerosis of nephron structures represent the common final pathway of histological renal lesions. These modifications are present in glomeruli and tubules. Sometimes the process of fibrosis affects predominantly either the glomeruli or the interstitium, suggesting different fibrosis mechanisms. In the end, nephron structures are replaced by fibrous and sclerosis tissue, leading to nephrosclerosis, the histopathological substrate of terminal chronic kidney disease.

This description suggests a homogenous process of fibrosis of the kidney structures (glomeruli, vessels, tubules and interstitium), but this is not an accurate representation. In the glomeruli at least, there are different pathways leading to various intensities of the fibrosis. So, hypertensive glomerulosclerosis associates a smaller glomerular volume due to ischemia when compared to FSGS. On the other hand, in diabetic nephropathy, the glomerular volume is high even in advanced stages of glomerulosclerosis.

Glomerulosclerosis is the process by which glomerular structures are replaced by fibrous tissue. There is a remarkable analogy with the process of atherosclerosis, despite structural difference. So, the functional similarity of mesangial cells with pericytes, the endothelial activation, macrophage infiltration, cell proliferation and the effect of the lipids, are all involved in both atherosclerosis and glomerulosclerosis.

The pioneers of nephrology have foreseen the changes that appear in the evolution of chronic kidney failure and their progressive character. They were describing the compensation mechanism through polyuria, systemic hypertension and high values of urea and creatinine. These induce a rise in the function of remaining nephrons. Although Bright already observed that protein restriction improves uremic symptoms, Addis suggested that its effect is produced by the reduction of filtration charge in the remaining nephrons and the improvement of chronic kidney insufficiency progression.

Nevertheless, understanding the way in which the chronic kidney failure progresses is the result of experimental models and therapeutic strategies. These have involved systemic and glomerular hypertension, glomerular hyper filtration and hypertrophy, podocyte lesions and proteinuria. In the end, these mechanisms activate cell signaling pathways leading to an disequilibrium between the production and the degradation of the extracellular matrix and the modification of its composition and also between cell proliferation and apoptosis. These mechanisms create the premises for the reversibility of renal lesions.

The model of 5/6 nephrectomy, reflecting the behaviour of healthy kidney and the micropunction studies have shown the elevation of SNGFR (single nephron glomerular filtration rate) as the consequence of glomerular hypertension and hyper filtration. The attempt to lower or to raise glomerular pressure proved their effect of inducing fibrosis. The administration of corticotherapy, ESAs and alimentary hyperproteic intake accelerate the evolution of renal fibrosis through the elevation of glomerular pressure. On the other hand, ACEs reduce the fibrosis by lowering glomerular pressure. Protein restriction also reduces the process of fibrosis in experimental ablative models by lowering glomerular pressure, raising GFR and 


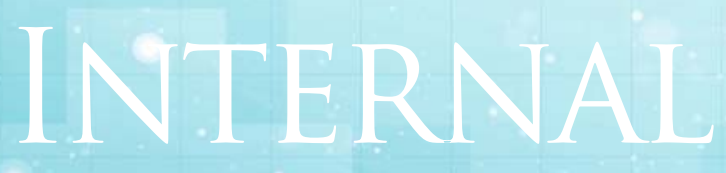

\section{General Reviews}

maintaining SNGFR, regardless of the arterial pressure.

In toxic nephritis through administration of puromycin, histopathologic findings suggest the fibrotic role of epithelial glomerular cells. In this model, the podocyte lesions induce the evolution towards GSFS. The morphologic and functional integrity of podocyte is important being a modulator of mesangial and endothelial cells proliferation and the main source of VEGF, angiopoietin and specific endothelial mitogen involved in capillary permeability and vascular structure organization.

Mutations of the genes implicated in nephrotic familial syndrome also highlight the importance of podocyte morphologic integrity. The podocyte is a terminal differentiated cell. The reduction of podocyte numbers produces fibrosis. I's interesting to note that lesions of the podocyte induce mesangial and endothelial lesions. Also, reduction of the podocyte number determinates area of glomerular membrane denudations and the free passage of plasmatic proteins which trigger fibrotic reaction.

Besides the adaptive mechanisms, the genetic factors are also involved in chronic kidney disease induction and progression. Different populations have different responses to the same mechanism of progression, sometimes more severe as is the case of Afro-Americans. For instance, there are genetic polymorphism of renin angiotensin aldosterone system having an accelerating role in renal fibrosis induction.

Renal mass at birth has also could effect on the appearance and progression of CKD. There is a correlation between renal mass and the number of nephrons at birth.

The therapeutic control of the mechanisms of progression of renal insufficiency have demonstrated that it could be possible to attenuate or even to stop the evolution towards renal fibrosis. Furthermore, the correction of physiologic disturbances have also improved the evolution of renal disease. Renin angiotensin aldosterone system activation is one of the most important mechanism of glomerular hypertension and also of glomerular fibrosis. RAAs is found in the glomerulus and in the macrophages eventually infiltrating the glomerulus. Angiotensin $\mathrm{II}$, besides being a potent hypertension inductor has many fibrotic effects directly or through aldosterone upregulation. Angiotensin II activates some cytokines which have potent fibrotic action like TGF-beta, PDGF, basic FGF, through induction of cell proliferation and extracellular matrix deposition.

Angiotensin II also upregulates PAI-1, an important modulator of proteolysis and ECM turnover. PAI-1 has been shown to be directly involved in glomerular and interstitial 
fibrosis. Moreover, PAI-1 inhibitor could prevent or in some models of prevention or inversion the fibrosis process. Aldosterone has also direct fibrotic effect besides its sodium conservative effect. The control of RAA pathway activation could be done by 3 classes of drugs: Renin inhibitors, inhibitors of Angiotensin I converting enzyme (ACEI), blockers of angiotensin II receptor AT1.

ACEls decrease glomerular pressure by reducing efferent arteriole tonus and by increasing glomerular bradykinin, as a consequence of its degradation inhibition. The blockers of Angiotensin II receptor AT1 do not have very intense effect of glomerular pressure reduction but they live unblocked Angiotensin II receptors AT1, which are involved in injured cells apoptosis and in reducing growth factors effect. Association of both drugs have important antiproteinuric effect and reduces fibrosis. Regarding arterial hypertension control, the association is not superior to any medication alone. Complete blocking of Angiotensin II is not possible due to other proteolytic systems, like chymotrypsin that could function as converting enzyme.

Many cytokines were involved in renal fibrosis. TGF-beta plays an important role due to its fibrotic effect and to immunological modulation, being at the crossway between immune and non-immune mechanisms. Transgenic animals for TGF-beta develop progressive kidney disease. Many fibrosis mechanisms are involved like increased ECM synthesis, phenotype transition of epithelial glomerular and tubular cells to a fibroblastic type, induction of podocytes apoptosis. TGFbeta action is biphasic, low concentrations being fibrotic and high concentrations inducing inflammation. The absence of TGF beta does not inhibit fibrosis. Instead, it induces lymphoproliferative syndromes due to the absence of regulatory immune action of the cytokine.

One of the drugs used in diabetes treatment could have antifibrotic actions as was suggested in some non-diabetic nephropathy models. These are thiazolidinedione. They activate a transcription factor, activator of peroxisome proliferator (PAR-gamma), involved in upregulation of some fibrotic cytokines like TGF- beta or proteolytic enzymes PAI-1.

Proteinuria, a consequence of morphological injury of glomerular membrane is known as accelerating renal fibrosis. The glomerular disease with high proteinuria progress more rapidly to end stage renal disease. The risk of mortality in renal insufficiency is stratified by the level of proteinuria. The toxic action of proteinuria is more evident to the tubules cells where it induces an inflammatory phenotype through NF-kB activation, upregulation of Angiotensin II and TGF-beta and epithelial-to mesenchymal transition. It seems rather the transfer of the glomerular injury to the interstitial space, where it induces fibrosis. Sometime interstitial fibrosis does not follow the glomerular one. This suggests that there are different mechanisms of fibrosis from those involved in glomeruli, starting with aglomerular tubules, tubulotoxic proteinuria, vascular lesions and tubular responses to immune mechanisms.

This is illustrated in diabetic nephropathy, where the retraction through fibrosis in the glomeruli coexists with interstitial expansion.

Returning to the initial idea of the reversibility of the injuries in diabetic nephropathy using pancreatic transplant, it seems that abnormal cellular insulin signalling, determines a specific response adapted to the reduced glucose use as a 


\section{INTERNAL}

\section{General Reviews}

source of energy, with consequences on cellular proliferation and extracellular matrix synthesis. Re-establishing insulin signalling restores cellular response and reverses fibrotic lesions.

The evolution of kidney failure is progressive due to the adaptive mechanisms to the reduction of the number of the nephrons. Therefore, it follows that reducing the activity of the organism to the level of the kidney functionality the duration of the evolution of the kidney failure could lengthen. That is often seen in patients with end-stage kidney failure that have never had any symptom. And as one of the main function of the kidney is the maintaining of body homeostasis, it appears that the diet of the patient is extremely important for the progression of the disease.

The associated pathophysiological mechanisms, activated by the failure of the homeostatic functions of the kidney, can directly affect the evolution of the disease. So, high blood pressure represents an adaptive mechanism to the kidney failure. It has to be noted that the kidney has the capacity to adapt to high blood pressure and only when this adaptive mechanisms are damaged the renal structures suffers from high blood pressure. For this reason, correctly controlling the blood pressure can reduce the kidney damage. The control of the patient's anaemia is also important, the kidney being responsible for the release of erythropoietin as a result of the decreasing oxygen tension in the tissues HIF signalling not only induces erythropoietin increase, but has also metabolic effects that tend to adapt cellular metabolism to the decrease of oxygen offer. The optimum level of haemoglobin has variated throughout the years. It depends on maintaining renal rheological conditions so that the tideresistance is minimal, but also depends on cardiac output and effective circulating volume.

The control of the phosphate-calcium metabolism is also important. Prevention of hyperphosphatemia and hypocalcaemia, D3 vitamin deficit, hyperparathyroidism, FGF 23 increase, control the cellular membrane excitability, vascular calcification and its haemodynamic consequences.

Concluding, controlling the kidney failure mechanisms can become a tool to rehabilitate these patients. The idea of substituting the kidney function itself it is also a method to rehabilitate these patients.

\section{References}

1.Olson JL, Heptinstall RH (1988) Nonimmunologic mechanisms of glomerular injury. Lab Invest 59:564578

2.Morrison AB, Howard RM (1966) The functional capacity of hypertrophied nephrons: effect of partial nephrectomy on the clearance of inulin and $P A H$ in the rat. J Exp Med 123:829844 5.

3.Shimamura T, Morrison AB (1975). A progressive 
glomerulosclerosis occurring in partial five-sixths nephrectomized rats. AmJ Pathol 79:95106

4.Brenner BM, Meyer TW, Hostetter TH (1982) Dietary protein intake and the progressive nature of kidney disease: the role of hemodynamically mediated glomerular injury in the pathogenesis of progressive glomerular sclerosis in aging, renal ablation, and intrinsic renal disease. N EnglJ Med 307:652659

5.Nath KA, Kren SM, Hostetter TH (1986) Dietary protein restriction in established renal injury in the rat. Selective role of glomerular capillary pressure in progressive glomerular dysfunction. J Clin Invest 78:11991205

6.Diamond JR, Karnovsky MJ; (1988) Focal and segmental glomeruloscierosis: Analogies to atherosclerosis; Kidney Int, 33. 9/7924
7.Fogo $A B$ (2000) Glomerular hypertension, abnormal glomerular growth, and progression of renal diseases. Kidney Int Suppl 75: S1521

8.Klahr S, (1990) Progression of chronic renal disease; Nutrition., 6(3):207-212.

9.Shankland SJ. 2006 The podocyte's response to injury: role in proteinuria and glomerulosclerosis. Kidney Int. 69(12):2131-47.

10. Nagata M. 2016 Podocyte injury and its consequences. Kidney Int. 89(6):1221-30

11.Lee HS, 2012 Mechanisms and consequences of TGFB overexpression by podocytes in progressive podocyte disease. Cell Tissue Res. 347(1):129-40

12.Fogo AB, 2007, Mechanisms of progression of chronic kidney disease; Pediatr Nephrol 22:20112022 